ITEP-TH-03/10

\title{
Quantum corrections from a path integral over reparametrizations
}

\author{
Yuri Makeenko* \\ Institute of Theoretical and Experimental Physics \\ B. Cheremushkinskaya 25, 117218 Moscow, Russia ${ }^{\dagger}$ \\ Poul Olesen* \\ The Niels Bohr International Academy, The Niels Bohr Institute \\ Blegdamsvej 17, 2100 Copenhagen Ø, Denmark
}

(Dated: November 20, 2018)

\begin{abstract}
We study the path integral over reparametrizations that has been proposed as an ansatz for the Wilson loops in the large- $N$ QCD and reproduces the area law in the classical limit of large loops. We show that a semiclassical expansion for a rectangular loop captures the Lüscher term associated with $d=26$ dimensions and propose a modification of the ansatz which reproduces the Lüscher term in other dimensions, which is observed in lattice QCD. We repeat the calculation for an outstretched ellipse advocating the emergence of an analog of the Lüscher term and verify this result by a direct computation of the determinant of the Laplace operator and the conformal anomaly.
\end{abstract}

PACS numbers: 11.15.Pg, 11.25.Tq, 12.38.Aw

\footnotetext{
*Also at the Institute for Advanced Cycling, Blegdamsvej 19, 2100 Copenhagen Ø, Denmark

$\dagger$ Electronic address: makeenko@itep.ru

${ }^{\ddagger}$ Electronic address: polesen@nbi.dk
} 


\section{INTRODUCTION}

Quantum fluctuations of surfaces are important in many physical phenomena. It is less known these fluctuations can sometimes be expressed in term of a functional integral over reparametrizations of variables relevant in the Feynman path integral. In this paper we shall consider the expression

$$
W(C) \equiv \int \mathcal{D} \theta \mathrm{e}^{-K A[x(\theta)]}
$$

where $x(\theta)$ is the boundary curve $C$ and where

$$
A[x(\theta)]=\frac{1}{8 \pi} \int_{0}^{2 \pi} \mathrm{d} \sigma \int_{0}^{2 \pi} \mathrm{d} \sigma^{\prime} \frac{\left(x(\theta(\sigma))-x\left(\theta\left(\sigma^{\prime}\right)\right)\right)^{2}}{1-\cos \left(\sigma-\sigma^{\prime}\right)} .
$$

The functional (2) is known in mathematics as the Douglas integral [1], whose minimum with respect to variations of the reparametrizations $\theta$ gives the minimal area

$$
A\left[x\left(\theta_{*}\right)\right]=S_{\min }(C) .
$$

Here $\theta_{*}(\sigma)$ is the saddle point of the integral (1). The functional integration (1) thus gives the area law to leading order.

The path integral over reparametrizations (1) was introduced in this context in Ref. [2] in connection with an off-shell string propagator. More recently it was proposed by Polyakov [3] as an ansatz for the Wilson loop in large $N$ QCD. The leading behavior obviously gives the leading behavior of the Wilson loop, found in most string models, where the bulk field $X^{\mu}(\tau, \sigma)$ satisfies the Dirichlet boundary condition

$$
\left.X^{\mu}(\tau, \sigma)\right|_{\text {boundary }}=x^{\mu}(\sigma) \text {. }
$$

To derive Eq. (3) from the Nambu-Goto action one can follow Douglas [1], or the more recent elegant paper by Migdal [4].

The functional integral (1) can be expanded around the saddle point $\theta_{*}$. With $\theta(\sigma)=$ $\theta_{*}(\sigma)+\beta(\sigma)$ we obtain the first non-vanishing contribution

$$
A_{2}=\frac{K}{8 \pi} \int_{0}^{2 \pi} \mathrm{d} \theta \int_{0}^{2 \pi} \mathrm{d} \theta^{\prime} \dot{x}(\theta) \cdot \dot{x}\left(\theta^{\prime}\right) \frac{\left(\beta(\theta)-\beta\left(\theta^{\prime}\right)\right)^{2}}{1-\cos \left(\sigma_{*}(\theta)-\sigma_{*}\left(\theta^{\prime}\right)\right)}
$$

Here $\sigma_{*}(\theta)$ is the inverse function of $\theta_{*}(\sigma)$.

Some dynamical consequences of Eq. (5) have been discussed by Rychkov [5] and by the authors [6]. However, it is perhaps fair to say that the physical meaning of the fluctuation integral (5) is not so clear. 
In this paper we shall show that the leading part of the fluctuations of large loops from (5) are transverse fluctuations of the minimal surface embedded by the curve $x\left(\theta_{*}\right)$. This we have shown for a rectangle and an ellipse, but we suspect the result to be more general.

To explain our result we mention that in the Nambu-Goto action transverse fluctuations add a contribution

$$
-\frac{d-2}{2} \operatorname{tr} \log \left(-\partial^{2}\right)=\frac{(d-2) \pi}{24} \frac{T}{R}
$$

to the area term for a large $R \times T$ rectangle with $T \gg R$. These quantum fluctuations (6) are called the Lüscher term [7]. By lattice Monte Carlo calculations in three and four dimensions, this term has been found to occur [8] in quenched $S U(N)$ for various $N^{\prime}$ s. Thus, for large distances the two leading terms from the Nambu-Goto action describe QCD quite well. Therefore, for a rectangular boundary curve the $T / R$ term can be identified with transverse fluctuations of the minimal surface.

With these remarks in mind we now give our main result: after integration over the fluctuations $\beta$ in the functional integral

$$
\int \mathcal{D} \beta \mathrm{e}^{-A_{2}}
$$

we obtain as the leading contribution the Lüscher term corresponding to $d=26$. In general, we obtain the $d$-dimensional contribution (plus the area term) from

$$
\int \mathcal{D} \theta \mathrm{e}^{-K A[x(\theta)]}(\operatorname{det} O)^{-(d-26) / 48} .
$$

Here $O$ is the operator which emerges in the semiclassical expansion of $A[x(\theta)]$ to quadratic order as exhibited in Eq. (5). This modification of Eq. (1) does not effect the classical limit leading to the area law, and has the meaning of a pre-exponential in the semiclassical approximation. Thus, this equation gives the leading effective QCD string behavior in terms of functional integration over reparametrizations.

We mention that our considerations may have potential applications in condensed matter. For example, in the inverse square XY model one encounters expressions somewhat similar to Eq. (5), see for example Ref. [9]. We shall, however, not pursue this track in the present paper.

The plan of this paper is the following: in Sect. II we discuss the general framework for the semiclassical approximation, and in Sect. III we carry out the functional integral over reparametrizations for a rectangle. A similar calculation for an ellipse is done in Sect. IV. 
In Sect. V we derive the generalization given by Eq. (8) and in Sect. VI we make some conclusions. A number of more technical points have been discussed in some Appendices.

\section{A SEMICLASSICAL CORRECTION}

The path integral over reparametrizations (1) reproduces the exponential of the minimal area in the classical limit $K S_{\min } \rightarrow \infty$. To calculate the semiclassical correction, we expand

$$
\theta(\sigma)=\theta_{*}(\sigma)+\beta(\sigma)
$$

or

$$
\sigma(\theta)=\sigma_{*}(\theta)+\beta(\theta)
$$

where

$$
\beta(0)=\beta(2 \pi)=0
$$

and expand the Douglas integral to quadratic order in $\beta$ around the classical trajectory $\theta_{*}(\sigma)$. This expansion makes sense because typical trajectories in the path integral over reparametrizations (1) are smooth as $K S_{\min } \rightarrow \infty$ and have Hausdorff dimension one [10].

Substituting (9) into Douglas' integral (2) and expanding in $\beta$, we find that the linear term vanishes because $\theta_{*}(\sigma)$ is the minimum, while the quadratic part reads

$$
A_{2}[\beta(\theta)]=\frac{K}{8 \pi} \int_{0}^{2 \pi} \mathrm{d} \theta_{1} \int_{0}^{2 \pi} \mathrm{d} \theta_{2} \dot{x}\left(\theta_{1}\right) \cdot \dot{x}\left(\theta_{2}\right) \frac{\left(\beta\left(\theta_{1}\right)-\beta\left(\theta_{2}\right)\right)^{2}}{1-\cos \left(\sigma_{*}\left(\theta_{1}\right)-\sigma_{*}\left(\theta_{2}\right)\right)} .
$$

The function $\beta(\theta)$ obeys

$$
\dot{\beta}(\theta) \geq-\dot{\sigma}_{*}(\theta)
$$

for the derivative of the reparametrizing function to be positive. This is always satisfied if $\beta$ is small and smooth enough.

In order to calculate the semiclassical correction to the area law, we need to do the Gaussian integral

$$
\int \mathcal{D} \beta(\theta) \mathrm{e}^{-A_{2}[\beta(\theta)]}
$$

with $A_{2}[\beta(\theta)]$ given by Eq. (12).

The typical values of $\beta$, which are essential in the path integral over $\beta$ in (14), are $\beta \sim 1 / \sqrt{K S_{\min }}$, i.e. small for $\sqrt{K S_{\min }} \gg 1$. Hence, the higher terms of an expansion 
of $A\left[\theta_{*}(\sigma)+\beta\right]$ in $\beta$ are suppressed [5] at large $\sqrt{K S_{\min }}$. The loop expansion goes in the parameter $1 / K S_{\min }$ and only one loop contributes with the given accuracy.

A comment is needed about the measure for the integration over $\beta(\theta)$. As is explained in Appendix A, the measure for the integration over $\sigma(\theta)$ involves a factor

$$
\frac{1}{\sigma^{\prime}}=\frac{1}{\sigma_{*}^{\prime}+\beta^{\prime}}=\frac{1}{\sigma_{*}^{\prime}}-\frac{\beta^{\prime}}{\left(\sigma_{*}^{\prime}\right)^{2}}+\frac{\left(\beta^{\prime}\right)^{2}}{\left(\sigma_{*}^{\prime}\right)^{3}}+\mathcal{O}\left(\left(\beta^{\prime}\right)^{3}\right) .
$$

Because $\beta \sim 1 / \sqrt{K S_{\min }}$, the second and third terms on the right-hand side are not essential to the given order. Therefore, the measure for the path integration over $\beta(\theta)$ in Eq. (14) is the usual one for smooth functions $\beta(\theta)$, while this factor will show up to the next order in $1 / K S_{\min }$

\section{PATH INTEGRAL OVER REPARAMETRIZATIONS: RECTANGLE}

In this section we show how the path integral over reparametrizations captures the Lüscher term for a rectangle.

The conformal map of the upper half plane onto the interior of a rectangle is given by the Schwarz-Christoffel mapping

$$
\omega=A F\left(\frac{z}{\sqrt{\mu}}, \mu\right)-\mathrm{i} \frac{A K\left(\sqrt{1-\mu^{2}}\right)}{2},
$$

where

$$
F\left(\frac{z}{\sqrt{\mu}}, \mu\right)=\int_{0}^{z} \frac{\mathrm{d} x}{\sqrt{\mu-x^{2}} \sqrt{1-\mu x^{2}}}
$$

is the incomplete elliptic integral of the first kind. The two parameters $A$ and $\mu$ are related to the coordinates of the vertices of the rectangle by

$$
A K(\mu)=\frac{R}{2}, \quad A K\left(\sqrt{1-\mu^{2}}\right)=T
$$

where $K(\mu)=F(1, \mu)$ is the complete elliptic integral of the first kind. In deriving Eq. (18) we used the important identity

$$
F(1 / \mu, \mu)=K(\mu)+\mathrm{i} K\left(\sqrt{1-\mu^{2}}\right) .
$$

Equations (18) relates $\mu$ to the ration of $R / T$ as

$$
\frac{2 T}{R}=\frac{K\left(\sqrt{1-\mu^{2}}\right)}{K(\mu)} .
$$


In the limit $T / R \rightarrow \infty$, when $\mu \rightarrow 0$, this equation simplifies to

$$
\pi \frac{T}{R}=\ln \frac{4}{\mu}
$$

When $z=s$ runs along the real axis, the variable $\omega$ runs along the boundary of the rectangle with $s=-1 / \sqrt{\mu},-\sqrt{\mu},+\sqrt{\mu},+1 / \sqrt{\mu}(\mu<1)$ mapped, respectively, onto the vertices of the rectangle: $(-R / 2, T / 2),(-R / 2,-T / 2),(R / 2,-T / 2),(R / 2, T / 2)$. The given choice of the argument of the mapping preserves the symmetry $s \rightarrow 1 / s$.

When $z$ has positive imaginary part, the coordinates

$$
X_{1}(z)=A \operatorname{Re} F\left(\frac{z}{\sqrt{\mu}}, \mu\right), \quad X_{2}(z)=A \operatorname{Im} F\left(\frac{z}{\sqrt{\mu}}, \mu\right)-\frac{A K\left(\sqrt{1-\mu^{2}}\right)}{2}
$$

take their values inside the rectangle. These coordinates are conformal. For this reason we have

$$
x_{1}\left(t_{*}(s)\right)=A \operatorname{Re} F\left(\frac{s}{\sqrt{\mu}}, \mu\right), \quad x_{2}\left(t_{*}(s)\right)=A \operatorname{Im} F\left(\frac{s}{\sqrt{\mu}}, \mu\right)-\frac{A K\left(\sqrt{1-\mu^{2}}\right)}{2},
$$

whose implementation for the function $t_{*}(s)$ is discussed below.

The boundary contour given by Eq. (23) satisfies Douglas' minimization (see Appendix B, Eq. (B3)). Correspondingly, the Douglas integral

$$
\frac{1}{4 \pi} \int_{-\infty}^{+\infty} \mathrm{d} s \int_{-\infty}^{+\infty} \mathrm{d} s^{\prime} \frac{\left[x\left(t_{*}\left(s_{1}\right)\right)-x\left(t_{*}\left(s_{2}\right)\right)\right]^{2}}{\left(s-s^{\prime}\right)^{2}}=2 A^{2} K(\mu) K\left(\sqrt{1-\mu^{2}}\right)=R T
$$

as it should. We have verified these two equations numerically.

A natural parametrization of the boundary of a rectangle is through $\tau \in S^{1}$ :

$$
\begin{array}{rlrl}
x_{1} & =\frac{T}{2} \tan \tau, \quad x_{2}=-\frac{T}{2} & & -\arctan \frac{R}{T} \leq \tau \leq \arctan \frac{R}{T} \\
x_{1} & =\frac{R}{2}, \quad x_{2}=-\frac{R}{2} \cot \tau & & \arctan \frac{R}{T} \leq \tau \leq \pi-\arctan \frac{R}{T} \\
x_{1} & =\frac{T}{2} \tan \tau, \quad x_{2}=\frac{T}{2} & \pi-\arctan \frac{R}{T} \leq \tau<\pi
\end{array}
$$

and analogously for negative $\tau$. Introducing

$$
t=\tan \frac{\tau}{2}
$$

we rewrite Eq. (25) as

$$
\begin{array}{rlrl}
x_{1}=T \frac{t}{1-t^{2}}, \quad x_{2}=-\frac{T}{2} & & -\frac{\sqrt{T^{2}+R^{2}}-T}{R} \leq t \leq \frac{\sqrt{T^{2}+R^{2}}-T}{R} \\
x_{1}=\frac{R}{2}, \quad x_{2}=R \frac{t^{2}-1}{4 t} & \frac{\sqrt{T^{2}+R^{2}}-T}{R} \leq t \leq \frac{\sqrt{T^{2}+R^{2}}+T}{R} \\
x_{1}=T \frac{t}{t^{2}-1}, \quad x_{2}=\frac{T}{2} & \frac{\sqrt{T^{2}+R^{2}}+T}{R} \leq t<+\infty .
\end{array}
$$


To relate $t$ to $s$, we identify

$$
\begin{gathered}
\frac{R}{2 K(\mu)} F\left(\frac{s}{\sqrt{\mu}}, \mu\right)=T \frac{t}{1-t^{2}} \\
\text { for }-\sqrt{\mu} \leq s \leq \sqrt{\mu} \quad \text { or } \quad-\frac{\sqrt{T^{2}+R^{2}}-T}{R} \leq t \leq \frac{\sqrt{T^{2}+R^{2}}-T}{R} \\
\frac{R}{2 K(\mu)} \int_{\sqrt{\mu}}^{s} \frac{\mathrm{d} x}{\sqrt{x^{2}-\mu} \sqrt{1-\mu x^{2}}}-\frac{T}{2}=R \frac{t^{2}-1}{4 t} \\
\text { for } \sqrt{\mu} \leq s \leq 1 / \sqrt{\mu} \quad \text { or } \quad \frac{\sqrt{T^{2}+R^{2}}-T}{R} \leq t \leq \frac{T+\sqrt{T^{2}+R^{2}}}{R} .
\end{gathered}
$$

Solving the quadratic equation for $t$ versus $s$, we obtain the minimizing function $t_{*}(s)$, which obviously obeys the boundary condition

$$
t(0)=0 .
$$

The symmetry $s \rightarrow 1 / s$ plays apparently an important role. It guarantees that the points $-\infty,-1,0,+1,+\infty$ are mapped onto themselves under the reparametrization $s \rightarrow t_{*}(s)$ : $t_{*}(-\infty)=-\infty, t_{*}(-1)=-1, t_{*}(0)=0, t_{*}(+1)=+1, t_{*}(+\infty)=+\infty$.

It is convenient to invert Eq. (28) using the Jacobi elliptic functions. Inverting Eq. (28a), we get

$$
\begin{gathered}
s=\sqrt{\mu} \operatorname{sn}\left(\frac{2 K(\mu) T}{R} \frac{t}{1-t^{2}}, \mu\right) \\
\text { for }-\sqrt{\mu} \leq s \leq \sqrt{\mu} \quad \text { or }-\frac{\sqrt{T^{2}+R^{2}}-T}{R} \leq t \leq \frac{\sqrt{T^{2}+R^{2}}-T}{R} .
\end{gathered}
$$

The function sn has a nice trigonometric expansion in the parameter (nome) (Ref. [11], $8.146 .1)$

$$
\exp \left(-\pi \frac{K\left(\sqrt{1-\nu^{2}}\right)}{K(\nu)}\right) \approx(\mu / 4)^{2} \ll 1
$$

giving

$$
s \approx \sqrt{\mu} \sin \left(\frac{\pi T}{R} \frac{t}{\left(1-t^{2}\right)}\right) .
$$

This formula is applicable for $-R / 2 T<t<+R / 2 T$, when $-\sqrt{\mu}<s<+\sqrt{\mu}$.

We can proceed in the same way with Eq. (28b), whose inverse is

$$
s=\sqrt{\mu} \mathrm{sn}\left(K(\mu)+\mathrm{i} K(\mu)\left(\frac{t^{2}-1}{2 t}+\frac{T}{R}\right), \mu\right) .
$$

Using the addition formula $([11], 8.156 .1)$ and the reduction of $\operatorname{sn}(x, 0)$ and $\operatorname{cn}(x, 0)$ to $\sin x$ and $\cos x$, we obtain

$$
s \approx \sqrt{\mu} \cosh \left[\frac{\pi}{4}\left(t-t^{-1}+\frac{2 T}{R}\right)\right] .
$$


However, this expansion is useless for large $t \rightarrow 2 T / R$, due to the imaginary part of the argument of sn the expansion will involve hyperbolic functions with arguments that can be large. Instead we can use the expansion of sn in terms of inverse sines ([11], 8.147.1), where these sines can be large, so only the first term is relevant:

$$
s \approx \frac{1}{\sqrt{\mu} \sin \left(\frac{\pi}{2}+\mathrm{i} \frac{\pi}{4}\left(\frac{t^{2}-1}{t}-\frac{2 T}{R}\right)\right)}=\frac{1}{\sqrt{\mu} \cosh \left[\frac{\pi}{4}\left(t-t^{-1}-\frac{2 T}{R}\right)\right]} .
$$

Equation (34) is applicable for $t \rightarrow R / 2 T$ from above and Eq. (35) is applicable when $t \rightarrow 2 T / R$.

The quadratic action, describing Gaussian fluctuations around $t_{*}(s)$, is like (12) rewritten for the real-axis parametrization:

$$
A_{2}[\beta(t)]=\frac{K}{4 \pi} \int \mathrm{d} t_{1} \int \mathrm{d} t_{2} \frac{\dot{x}\left(t_{1}\right) \cdot \dot{x}\left(t_{2}\right)}{\left(s_{*}\left(t_{1}\right)-s_{*}\left(t_{2}\right)\right)^{2}}\left[\beta\left(t_{1}\right)-\beta\left(t_{2}\right)\right]^{2} .
$$

The Gaussian approximations is justified for large $K R T$, when

$$
\beta(t) \sim \frac{1}{\sqrt{K R T}} .
$$

To calculate the path integral over the quantum fluctuations around the minimizing function $t_{*}(s)$, we need a mode expansion of the infinitesimal reparametrizing function $\beta(t)$. To get rid of the projective symmetry, we keep fixed 3 points, e.g. $-1,0,+1$ or $-\sqrt{\mu}, 0, \sqrt{\mu}$ fixed: $\beta(-1)=0, \beta(0)=0, \beta(+1)=0$ or $\beta\left(-t_{*}(-\sqrt{\mu})\right)=0, \beta(0)=0, \beta\left(t_{*}(\sqrt{\mu})\right)=0$.

For each segment from $t_{i}$ to $t_{f}$, we consider the mode expansion

$$
\beta(t)=\sum_{n} c_{n} \sin \left(\pi n \frac{t-t_{i}}{t_{f}-t_{i}}\right),
$$

obeying the boundary condition $\beta\left(t_{i}\right)=\beta\left(t_{f}\right)=0$. This set of sines forms a complete basis on the given interval. Actually we shall need the mode expansion for 4 segments attached to $t=t_{*}( \pm \sqrt{\mu}) \approx \pm R / 2 T$ because the large contribution of $1 / \mu$ will appear in $A_{2}$ only for those.

The appearance of the large factor $1 / \mu$ is seen already from Eqs. (30), (33) because $s_{*}$ in the denominator in Eq. (36) is proportional to $\sqrt{\mu}$. We need, however, to show that this $1 / \mu$ is multiplied by a factor $\sim 1$.

Let us analyze the contribution to (36) that comes from $-R / 2 T<t_{1}, t_{2}<+R / 2 T$, i.e. from the bottom side of the rectangle. Introducing the variable

$$
y=\frac{2 T}{R} t, \quad-1<y<1
$$


and using Eq. (32), we write the contribution of this domain to $A_{2}$ as

$$
\frac{K T^{2}}{4 \pi \mu} \int_{-1}^{1} \mathrm{~d} y_{1} \int_{-1}^{1} \mathrm{~d} y_{2} \frac{\left(\beta\left(y_{1}\right)-\beta\left(y_{2}\right)\right)^{2}}{\left(\sin \left(\pi y_{1} / 2\right)-\sin \left(\pi y_{2} / 2\right)\right)^{2}} \propto \frac{T}{\mu R} C
$$

with a positive constant $C$. A similar contribution appears if $t_{1}-R / 2 T \sim t_{2}-R / 2 T \sim$ $R^{2} /(2 T)^{2}$ as is prescribed by Eq. (34) for both $t_{1}>R / 2 T$ and $t_{2}>R / 2 T$. An analogous contribution (with possibly some powers of $T / R \propto \ln (4 / \mu)$ ) emerges also when $t_{1}<R / 2 T$ and $t_{2}>R / 2 T$ or $t_{1}>R / 2 T$ and $t_{2}<R / 2 T$.

Therefore, the path integral over the quantum fluctuations around the minimizing function $t_{*}(s)$ gives, using the $\zeta$-function regularization and Eq. (21) (and disregarding the logs to the order in consideration)

$$
\prod_{\text {modes }} \sqrt{\mu} \propto\left(\frac{1}{\sqrt[4]{\mu}}\right)^{4} \propto \exp \left(\frac{\pi T}{R}\right),
$$

where the 4 th power is due to the four sets of modes ${ }^{1}$. This remarkably reproduces the Lüscher term $(6)$ in $d=26$ !

There was a subtlety in the derivation - the appearance of a logarithmic divergence at the corners of the rectangle if $\dot{\beta}$ (corners) $\neq 0$. It is seen from Eq. (40), where the region near $y_{1}=y_{2}=1$ or $y_{1}=y_{2}=-1$ (associated with $t_{1}=t_{2} \rightarrow R / 2 T$ or $t_{1}=t_{2} \rightarrow-R / 2 T$ ) produces the logarithmic divergence

$$
\int_{-1+\delta}^{1-\delta} \mathrm{d} y_{1} \int_{-1+\delta}^{1-\delta} \mathrm{d} y_{2} \frac{\dot{\beta}^{2}( \pm 1)}{\left(y_{1}+y_{2} \mp 2\right)^{2}}=\dot{\beta}^{2}( \pm 1) \ln \frac{1}{\delta}
$$

with the upper (lower) sign referring to $y=1(y=-1)$. The coefficient is nonvanishing if $\dot{\beta}( \pm 1) \neq 0$. Analogously, the integral is logarithmically divergent at the corner, when $t_{1}, t_{2}>R / 2 T$ or $t_{1}<R / 2 T, t_{2}>R / 2 T$ and vise versa.

The logarithmic divergence can be regularized by smoothing the corners like in Refs. [7, 14]. It is clear from such a regularization that the contribution of trajectories with $\dot{\beta}(R / 2 T) \neq 0$ to the path integral over $\beta(t)$ will be suppressed as the smoothing is removed. Consequently, this corner divergence does not effect the result of this section. In the next section we repeat the consideration for the case of an ellipse, when there are no corners.

If $d \neq 26$, the asymptotic ansatz for the Wilson loops has to be improved to get the correct factor $(d-2) / 24$ in the Lüscher term (6). This issue will be described in Sect. V.

\footnotetext{
1 This is like in the computation of the static potential for the Polyakov string in Ref. [12].
} 


\section{PATH INTEGRAL OVER REPARAMETRIZATIONS: ELLIPSE}

In this section we evaluate the path integral over reparametrizations for an ellipse and obtain a prediction for the associated Lüscher term.

The necessary formulas are given in Appendix B of [6] and are partially reproduced in Appendix B. We are interested in the case of a very long ellipse when the ratio $b / a \rightarrow 0$ and $\nu \rightarrow 1$ according to

$$
\ln \frac{a+b}{a-b}=\frac{\pi K\left(\sqrt{1-\nu^{2}}\right)}{2 K(\nu)}
$$

Using the asymptote

$$
K(\nu) \stackrel{\nu \rightarrow 1}{\rightarrow} \frac{1}{2} \ln \frac{8}{(1-\nu)}
$$

we simplify Eq. (43) to

$$
\frac{b}{a}=\frac{\pi^{2}}{4 \ln \frac{8}{(1-\nu)}}
$$

For $\nu \rightarrow 1$ the elliptic function simplifies and we have

$$
\theta_{*}(\sigma)=\pi\left(\frac{\ln \frac{2 \sigma+\sqrt{4 \sigma^{2}+(1-\nu)^{2}}}{8}}{\ln \frac{8}{(1-\nu)}}+1\right)
$$

for $-\pi / 2<\sigma<\pi / 2$. Inverting Eq. (46), we find

$$
\sigma_{*}(\theta)=\frac{1-\nu}{2} \sinh \left(\frac{2 \theta}{\pi} \ln \frac{8}{1-\nu}\right) .
$$

This is quite similar to Eqs. (32) and (34) for a rectangle with $\sqrt{\mu}$ replaced by $(1-\nu)$.

The calculation of the path integral over reparametrizations at one loop is quite analogous to that for the rectangle described in the previous section. We see that the large factor of $(1-\nu)^{-2}$ emerges in Eq. (12) because $\sigma_{*} \propto(1-\nu)$ from Eq. (47). To evaluate the coefficient, let us consider the domain of small $\theta_{1}$ and $\theta_{2}$ :

$$
\theta_{1}, \theta_{2} \ll\left(\ln \frac{8}{1-\nu}\right)^{-1}
$$

which contributes to the integral in (12)

$$
\frac{K}{(1-\nu)^{2}} \int \mathrm{d} \theta_{1} \int \mathrm{d} \theta_{2}\left(a^{2} \theta_{1} \theta_{2}+b^{2}\right) \frac{\dot{\beta}^{2}\left(\theta_{1}\right)\left(\theta_{2}-\theta_{1}\right)^{2}}{\left(\theta_{2}-\theta_{1}\right)^{2} \ln ^{2} \frac{8}{1-\nu}} \propto \frac{1}{(1-\nu)^{2}}
$$

modulo the powers of $b / a \propto\left(\ln \frac{8}{(1-\nu)}\right)^{-1}$. The same contribution comes also from the domain of both $\theta_{1}$ and $\theta_{2}$ near $\pi$. We thus have

$$
A_{2} \propto \frac{1}{(1-\nu)^{2}}
$$


for every mode.

Integrating the Gaussian integral for every mode and using the $\zeta$-function regularization, we get (disregarding the logs to this order) a pre-factor of the type

$$
\prod_{\text {modes }}(1-\nu) \propto\left(\frac{1}{\sqrt{1-\nu}}\right)^{4} \propto \exp \left(\frac{\pi^{2}}{2} \frac{a}{b}\right)
$$

where the product runs over 4 sets of modes, which results in a factor of 4 in the exponent. This coincides with the Lüscher term (6) for a rectangle of the size $R \times T$ in $d=26$ dimensions provided $^{2}$

$$
\frac{T}{R}=\frac{\pi a}{2 b}
$$

In Appendices $\mathrm{C}$ and $\mathrm{D}$ we confirm this by an explicit calculation of the determinant of the Laplace operator and the conformal anomaly for an outstretched ellipse.

\section{A GENERALIZATION TO ARBITRARY DIMENSIONS}

The results of two previous sections demonstrate the already mentioned fact that the ansatz (1) has to be modified in order to describe the Lüscher term in $d=4$ dimensions.

A simple modification is based on the form of the path integral for a rectangle

$$
\int \mathcal{D} \beta(t) \mathrm{e}^{-A_{2}[\beta(t)]} \stackrel{T \gg R}{\propto} \mathrm{e}^{\pi T / R}
$$

with quadratic action $A_{2}[\beta(t)]$ given by Eq. (36).

For an arbitrary curve this path integral can be expressed through the determinant of the corresponding operator, that enters $A_{2}$, which we denote as $O$ :

$$
\int \mathcal{D} \beta(t) \mathrm{e}^{-A_{2}[\beta(t)]}=(\operatorname{det} O)^{-1 / 2}
$$

It is now clear that the following modification of the ansatz (1) will provide the correct value of the Lüscher term (6) in $d$ dimensions:

$$
\int \mathcal{D} t \mathrm{e}^{-K A[x(t)]}(\operatorname{det} O)^{-(d-26) / 48}
$$

This modification of Eq. (1) does not effect the classical limit, leading to the area law, and has the meaning of altering a pre-exponential in the semiclassical approximation.

\footnotetext{
${ }^{2}$ It is worth noting that the Wilson loops for a rectangle and ellipse then coincide [13] in $d=4$ (the only dimension with a $T / R$ Coulomb term) to the second order of perturbation theory.
} 
To make the structure of the operator $O$ more explicit, it is convenient to use the expansion (9) of the direct function $\theta(\sigma)$ rather than that (10) of the inverse function $\sigma(\theta)$ as above. Using the identity

$$
\beta\left(t_{*}(s)\right)=-\frac{1}{\frac{\mathrm{d} t_{*}(s)}{\mathrm{d} s}} \beta(s),
$$

which stems from the definitions (9) and (10), we then obtain for the real-axis parametrization:

$$
A_{2}[\beta(s)]=\frac{K}{4 \pi} \int \mathrm{d} s_{1} \int \mathrm{d} s_{2} \frac{\dot{x}\left(t_{*}\left(s_{1}\right)\right) \cdot \dot{x}\left(t_{*}\left(s_{2}\right)\right)}{\left(s_{1}-s_{2}\right)^{2}}\left[\beta\left(s_{1}\right)-\beta\left(s_{2}\right)\right]^{2},
$$

which determines the "momentum" (with respect to $s$ ) space operator

$$
O\left(p_{1}, p_{2}\right)=\frac{K}{8 \pi} \int \mathrm{d} q|q|\left(2 \dot{x}\left(p_{1}+q\right) \cdot \dot{x}\left(-p_{2}-q\right)-\dot{x}\left(p_{1}-p_{2}+q\right) \cdot \dot{x}(-q)-\dot{x}(q) \cdot \dot{x}\left(p_{1}-p_{2}-q\right)\right)
$$

with

$$
\dot{x}(p) \equiv \int \mathrm{d} s \mathrm{e}^{\mathrm{i} p s} \dot{x}\left(t_{*}(s)\right) .
$$

We can finally substitute $t_{*}(s)$ by $t(s)$ in this formula without changing the semiclassical approximation.

It is worth noting that in contrast to the Laplace operator of Ref. [14], where the Lüscher term was obtained for the Polyakov string, the present operator $O$ lives in the boundary, which makes the construction nontrivial.

\section{CONCLUSIONS}

The conclusion is that the reparametrization of the boundary curve involved in Eq. (1) carries information on the transverse fluctuations in 26 dimensions. As is shown in the previous section, it is possible to generalize this to any dimensions.

Our motivation for the present paper is our previous work on the QCD/string scattering amplitudes [15], where we used that the amplitude can be expressed in terms of a Wilson loop through Feynman path integration. There we only considered the leading area behavior. However, having developed a path integral expression for the next term, we hope that the $x^{\mu}$ integrals can be performed, thereby providing a momentum space analogue of the Lüscher term. We hope this may help to answer a very interesting question as to how the intercept of the Regge trajectory changes under such a modification of the ansatz for the Wilson loops. 


\section{Acknowledgments}

We are indebted to Andrey Mironov and Niels Obers for useful discussions.

\section{Appendix A: Measure for integrating over reparametrization}

Introducing

$$
v_{i}=s_{i}-s_{i-1} \quad s_{N}=s_{f},
$$

we rewrite the measure of [6] for the integration over reparametrizations as

$$
\begin{aligned}
\int_{s_{0}}^{s_{f}} \mathcal{D}_{\mathrm{diff}} s & \equiv \lim _{N \rightarrow \infty} \prod_{i=2}^{N-1} \int_{s_{0}}^{s_{i+1}} \frac{\mathrm{d} s_{i}}{\left(s_{i+1}-s_{i}\right)} \int_{s_{0}}^{s_{2}} \frac{\mathrm{d} s_{1}}{\left(s_{2}-s_{1}\right)\left(s_{1}-s_{0}\right)} \\
& =\lim _{N \rightarrow \infty} \prod_{i=1}^{N} \int_{0}^{\infty} \frac{\mathrm{d} v_{i}}{v_{i}} \delta^{(1)}\left(s_{f}-s_{0}-\sum_{j=1}^{N} v_{j}\right) .
\end{aligned}
$$

The integration over $v_{i}$ 's in Eq. (A2) can be represented through the integration over a scalar field as follows. Writing

$$
v_{i}=\mathrm{e}^{\psi_{i} / 2}
$$

we have

$$
\int_{0}^{\infty} \frac{\mathrm{d} v_{i}}{v_{i}} \cdots=\frac{1}{2} \int_{-\infty}^{+\infty} \mathrm{d} \psi_{i}
$$

and

$$
\int_{s_{0}}^{s_{f}} \mathcal{D}_{\operatorname{diff}} s=\lim _{N \rightarrow \infty} \frac{1}{2^{N}} \prod_{i=1}^{N} \int_{-\infty}^{+\infty} \mathrm{d} \psi_{i} \delta^{(1)}\left(s_{f}-s_{0}-\sum_{j=1}^{N} \mathrm{e}^{-\psi_{j} / 2}\right) .
$$

This represents the continuous measure as

$$
\int_{s_{0}}^{s_{f}} \mathcal{D}_{\text {diff }} s=\int \mathcal{D} \psi \delta^{(1)}\left(s_{f}-s_{0}-\int_{s_{0}}^{s_{f}} \mathrm{~d} t \mathrm{e}^{-\psi(t) / 2}\right),
$$

where $t$ is a certain parametrization of the contour (e.g. through the proper time) and $\mathcal{D} \psi$ is the usual measure

$$
\int \mathcal{D} \psi=\prod_{s=s_{0}}^{s_{f}} \int_{-\infty}^{+\infty} \mathrm{d} \psi(s)
$$

The scalar field $\psi$, that appears in Eqs. (A1), (A3), is in fact a discretization of the boundary value of the Liouville field

$$
\left.\varphi(\tau, s)\right|_{\text {boundary }}=\psi(s)
$$


which is related to the boundary metric:

$$
\sqrt{\left(\frac{\mathrm{d} x^{\mu}(s)}{\mathrm{d} s}\right)^{2}}=\mathrm{e}^{\psi(s) / 2}
$$

Under the reparametrization $s \rightarrow f(s)(\mathrm{d} f / \mathrm{d} s \geq 0)$, the boundary value of the Liouville field $\psi(s)$ transforms as

$$
\psi(s) \rightarrow \psi(f(s))=\psi(s)-2 \ln \frac{\mathrm{d} f(s)}{\mathrm{d} s}
$$

that clarifies its relation to reparametrizations.

The results of this Appendix make it possible to relate the ansatz (1) with Eq. (17) of Ref. [14], where the path integral over $\psi$ is the same as the path integral over reparametrization in view of Eq. (11) of [14]. They coincide in $d=26$ provided $S_{\mathrm{cl}}$ is the Douglas integral.

\section{Appendix B: Douglas' algorithm for plane contours: conformal map}

The construction of the coordinates of the minimal surface, enclosed by a plane contour, is given by conformal mappings. Let us describe such a contour by two functions $x_{1}(t)$ and $x_{2}(t)$. Motivated by Appendix H of Ref. [4], we define the analytic functions

$$
\Phi_{\mu}(z)=\int_{-\infty}^{+\infty} \frac{\mathrm{d} s^{\prime}}{\pi} \frac{\left[x_{\mu}\left(t_{*}\left(s^{\prime}\right)\right)-x_{\mu}\left(t_{*}(z)\right)\right]}{\left(s^{\prime}-z\right)^{2}} .
$$

The real and imaginary parts of $\Phi_{\mu}$ are

$$
\begin{aligned}
& \operatorname{Re} \Phi_{\mu}(s)=\int_{-\infty}^{+\infty} \frac{\mathrm{d} s^{\prime}}{s^{\prime}-s} \dot{x}_{\mu}\left(t_{*}\left(s^{\prime}\right)\right) \\
& \operatorname{Im} \Phi_{\mu}(s)=\frac{\mathrm{d} x_{\mu}\left(t_{*}(s)\right)}{\mathrm{d} s}=\dot{x}_{\mu}\left(t_{*}(s)\right) \frac{\mathrm{d} t_{*}(s)}{\mathrm{d} s} .
\end{aligned}
$$

Therefore, Douglas' minimization equation

$$
\int_{-\infty}^{+\infty} \mathrm{d} s^{\prime} \frac{\dot{x}\left(t_{*}(s)\right) \cdot\left[x\left(t_{*}(s)\right)-x\left(t_{*}\left(s^{\prime}\right)\right)\right]}{\left(s-s^{\prime}\right)^{2}}=0 .
$$

is satisfied if

$$
\sum_{\mu} \operatorname{Im} \Phi_{\mu}^{2}(s)=0
$$

at the real axis.

For the circle we have

$$
x_{1}(t)+\mathrm{i} x_{2}(t) \equiv C(t)=\frac{\mathrm{i}-t}{\mathrm{i}+t}
$$


so Eq. (B1) gives

$$
\Phi_{1}(z)=\mathrm{i} \Phi_{2}(z)=\frac{2}{(\mathrm{i}+z)^{2}}
$$

with

$$
\sum_{\mu} \Phi_{\mu}^{2}(s)=0
$$

which obviously obeys Eq. (B4). ¿From Eqs. (B6) and (B5) we find

$$
\begin{aligned}
\operatorname{Im} \Phi_{1}(s) & =-\frac{2 s}{\left(1+s^{2}\right)^{2}}, & \frac{1}{2} \dot{x}_{1}(t) & =-\frac{2 t}{\left(1+t^{2}\right)^{2}} ; \\
\operatorname{Im} \Phi_{2}(s) & =\frac{1-s^{2}}{\left(1+s^{2}\right)^{2}}, & \frac{1}{2} \dot{x}_{2}(t) & =\frac{1-t^{2}}{\left(1+t^{2}\right)^{2}}
\end{aligned}
$$

and from Eq. (B2b) conclude that $t_{*}(s)=s$, as it should be for the circle.

For the functions obeying Eq. (B7), we always have $\Phi_{1}(s)=\mathrm{i} \Phi_{2}(s)$ and

$$
\begin{aligned}
& \Phi(s) \equiv \Phi_{1}(s)+\mathrm{i} \Phi_{2}(s)=2 \Phi_{1}(s) \\
& \operatorname{Im} \Phi_{1}(s)+\mathrm{i} \operatorname{Im} \Phi_{2}(s)=-\frac{\mathrm{i}}{2} \Phi(s) .
\end{aligned}
$$

Equation (B2b) can then be rewritten as

$$
-\frac{\mathrm{i}}{2} \Phi(s)=\frac{\mathrm{d} C\left(t_{*}(s)\right)}{\mathrm{d} s}=\dot{C}\left(t_{*}(s)\right) \frac{\mathrm{d} t_{*}(s)}{\mathrm{d} s} .
$$

For the given analytic function $C(z)$ it determines the reparametrizing function $t_{*}(s)$.

For the circle and ellipse it is more convenient to use the unit-disk parametrization, when

$$
\Phi(\omega)=(\omega+1)^{2} \oint_{S^{1}} \frac{\mathrm{d} \omega}{2 \pi \mathrm{i}} \frac{\left[C(\omega)-C\left(\omega^{\prime}\right)\right]}{\left(\omega-\omega^{\prime}\right)^{2}} .
$$

For the ellipse $C(\omega)$ is given by the conformal map (where $\nu$ is the same as $s$ in Eq. (B26) of $[6])$

$$
C(\omega)=\sqrt{a^{2}-b^{2}} \sin \left[\frac{\pi}{2 K(\nu)} F\left(\frac{\omega}{\sqrt{\nu}}, \nu\right)\right]
$$

and from Eq. (B11) we obtain

$$
\begin{aligned}
\Phi(\omega) & =\sqrt{a^{2}-b^{2}} \frac{\pi}{2 K(\nu)} \frac{(\omega+1)^{2}}{\sqrt{\nu-\omega^{2}} \sqrt{1-\nu \omega^{2}}} \cos \left[\frac{\pi}{2 K(\nu)} F\left(\frac{\omega}{\sqrt{\nu}}, \nu\right)\right] \\
& =\left(\omega^{2}+1\right) \frac{\mathrm{d} C(\omega)}{\mathrm{d} \omega} .
\end{aligned}
$$

The final step is to extract $\mathrm{d} \theta_{*}(\sigma) / \mathrm{d} \sigma$ from Eq. (B10). Remembering that

$$
C(\theta)=a \cos \theta+\mathrm{i} b \sin \theta,
$$

we find

$$
\frac{\mathrm{d} \theta}{\mathrm{d} \omega}=\frac{1}{\sqrt{\nu-\omega^{2}} \sqrt{1-\nu \omega^{2}}}
$$

which for $\omega=\mathrm{e}^{\mathrm{i} \sigma}$ reproduces Eq. (B32) of [6]. 


\section{Appendix C: Calculating the Lüscher term for an ellipse}

\section{Elliptical coordinates and Mathieu functions}

Let us consider an ellipse as the boundary contour. To calculate the determinant of the Laplace operator with this boundary conditions, we parametrize the surface by elliptical (Lamé) coordinates

$$
x_{1}=h \cosh \tau \cos \sigma, \quad x_{2}=h \sinh \tau \sin \sigma
$$

or

$$
x_{1}+\mathrm{i} x_{2}=h \cosh (\tau+\mathrm{i} \sigma) .
$$

Here $\sigma \in[0,2 \pi)$ and $\tau \in\left[0, \tau_{0}\right]$. The boundary is approached for $\tau=\tau_{0}=\operatorname{arctanh} b / a$ and foci are at $( \pm h, 0)$ with

$$
h=\sqrt{a^{2}-b^{2}} .
$$

The elliptic coordinates are conformal:

$$
\mathrm{d} s^{2}=h^{2}\left(\sinh ^{2} \tau+\sin ^{2} \sigma\right)\left(\mathrm{d} \tau^{2}+\mathrm{d} \sigma^{2}\right)
$$

so the area in the conformal gauge reads

$$
A=\frac{1}{2} \int_{0}^{\tau_{0}} \mathrm{~d} \tau \int_{0}^{2 \pi} \mathrm{d} \sigma\left(\frac{\partial X}{\partial \tau} \cdot \frac{\partial X}{\partial \tau}+\frac{\partial X}{\partial \sigma} \cdot \frac{\partial X}{\partial \sigma}\right)
$$

The Laplacian reads in elliptic coordinates as

$$
\Delta=\frac{1}{h^{2}\left(\sinh ^{2} \tau+\sin ^{2} \sigma\right)}\left(\frac{\partial^{2}}{\partial \tau^{2}}+\frac{\partial^{2}}{\partial \sigma^{2}}\right) .
$$

The Laplace equation separates in elliptic coordinates to the Mathieu equations

$$
\begin{gathered}
\frac{\mathrm{d}^{2} F}{\mathrm{~d} \sigma^{2}}+(\alpha-2 q \cos 2 \sigma) F=0 \\
\frac{\mathrm{d}^{2} G}{\mathrm{~d} \tau^{2}}-(\alpha-2 q \cosh 2 \tau) G=0 .
\end{gathered}
$$

where $\alpha$ is a separation constant and $q$ is related to the eigenvalue $\lambda$ by

$$
q=\lambda \frac{h^{2}}{4}
$$

A complete set of solutions for $F$ and $G$ is given, respectively, by the Mathieu functions $c e_{m}(\sigma, q), s e_{m}(\sigma, q)$ and the modified Mathieu functions $C e_{m}(\tau, q), S e_{m}(\tau, q)$ of integral order $m$. 


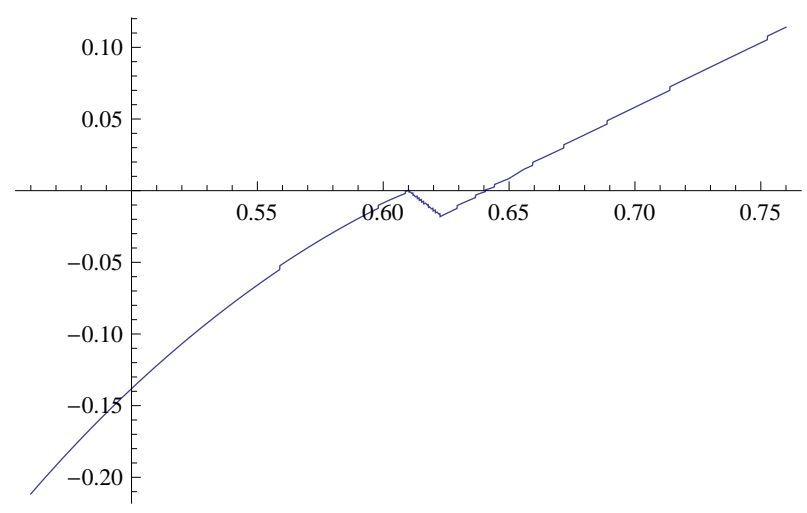

FIG. 1: Plot of $1-\alpha_{m}(q) / 2 q$ versus $c=q / m^{2}$.

While the characteristic numbers $\alpha_{m}(q)$ and $\beta_{m}(q)$ for the $c e_{m}$ and $s e_{m}$ modes are not explicitly known for large $q \sim m^{2}$, which would be the case for an outstretched ellipse with $b / a \ll 1$, two asymptotic formulas exist [16]

$$
\begin{aligned}
& \alpha_{m}=\beta_{m} \stackrel{m^{2} \gg q}{=} m^{2}+\frac{1}{2} \frac{q^{2}}{m^{2}}+\frac{5}{32} \frac{q^{4}}{m^{6}}+\frac{9}{64} \frac{q^{6}}{m^{10}}+\frac{1469}{8192} \frac{q^{8}}{m^{14}}+\frac{4471}{16384} \frac{q^{10}}{m^{18}}+\ldots \\
& \alpha_{m}=\beta_{m} \stackrel{m^{2} \ll q}{=}-2 q+4 m q^{1 / 2}-\frac{1}{2} m^{2}-\frac{1}{2^{4}} \frac{m^{3}}{q^{1 / 2}}-\frac{5}{2^{8}} \frac{m^{4}}{q}-\frac{33}{2^{12}} \frac{m^{5}}{q^{3 / 2}}-\frac{63}{2^{14}} \frac{m^{6}}{q^{2}}-\frac{527}{2^{18}} \frac{m^{7}}{q^{5 / 2}}-\ldots
\end{aligned}
$$

These expansions are not formally applicable for $c=q / m^{2} \sim 1,{ }^{3}$ but the first formula works numerically for $c \lesssim 0.7$, while second formula works numerically for $c \gtrsim 0.4-0.5$, so there is an overlap. Special values of $c$ are $c=0.61$ and $c=0.64$, when

$$
\alpha_{m}\left(\mathrm{~cm}^{2}\right)=\beta_{m}\left(\mathrm{~cm}^{2}\right)=2 \mathrm{~cm}^{2}=2 q
$$

They are obtained numerically with Mathematica, but are derivable from the expansions (C9a) and (C9b), correspondingly. As we see from Fig. 1, the difference $2 q-\alpha_{m}(q)$ is positive for $c>0.64$, where the expansion $(\mathrm{C} 9 \mathrm{~b})$ is applicable.

The boundary condition requires $C e_{m}\left(\tau_{0}\right)=0$ and $S e_{m}\left(\tau_{0}\right)=0$, which implies

$$
\tau_{0}=Z_{m, n}
$$

where $Z_{m, n}$ are the corresponding zeros. This determines the eigenvalues of the Laplacian with $m$ and $n$ playing the role of angular and radial quantum numbers, respectively. For

\footnotetext{
${ }^{3}$ Some results concerning the Mathieu characteristic numbers for large $q \sim m^{2}$ can be found in Ref. [17].
} 
small $\tau_{0}(=\operatorname{large} a / b)$ we have $[16]$

$$
\begin{aligned}
& C e_{m}(\tau) \approx \text { const. } \times \cos \left(\sqrt{2 q-\alpha_{m}} \tau\right) \\
& S e_{m}(\tau) \approx \text { const. } \times \sin \left(\sqrt{2 q-\beta_{m}} \tau\right)
\end{aligned}
$$

so that

$$
\sqrt{2 q-\alpha_{m}} \tau_{0}=\frac{\pi n}{2}
$$

because $\alpha_{m}=\beta_{m}$ for large $m$. Here $n$ is odd or even for the Ce or Se modes, respectively. We conclude therefore that

$$
2 q-\alpha_{m}=\left(\frac{\pi n a}{2 b}\right)^{2}
$$

has to be large and justify large $q \sim m^{2}$.

\section{Evaluating the determinant}

Each mode contributes

$$
\operatorname{det}\left(\Delta^{-1 / 2}\right)=\prod_{n, m} \lambda_{n, m}^{-1 / 2}=\mathrm{e}^{\sum_{n, m} \ln \left(\lambda_{n, m}^{-1 / 2}\right)} .
$$

Since $a$ is large, the sum over $m$ can be replaced by an integral over $\omega=m / a$ like in Ref. [18] and for large $q^{1 / 2} \sim m \sim a / b$, we have

$$
\lambda_{n, m}^{1 / 2}=\operatorname{rf}\left(\frac{\omega}{r}\right)
$$

where

$$
r=\frac{\pi n}{2 b}
$$

so that

$$
\sum_{m} \ln \left(\lambda_{n, m}^{-1 / 2}\right)=-a \int_{0}^{\infty} \mathrm{d} \omega \ln \left[r f\left(\frac{\omega}{r}\right)\right] .
$$

For large $r$ the integral on the right-hand side is proportional to $r$ and we can get the coefficient of proportionality by differentiating with respect to $r$. This gives

$$
\sum_{m} \ln \left(\lambda_{n, m}^{-1 / 2}\right)=-a r \int_{0}^{\infty} \mathrm{d} x\left[1-x f^{\prime}(x) / f(x)\right]
$$



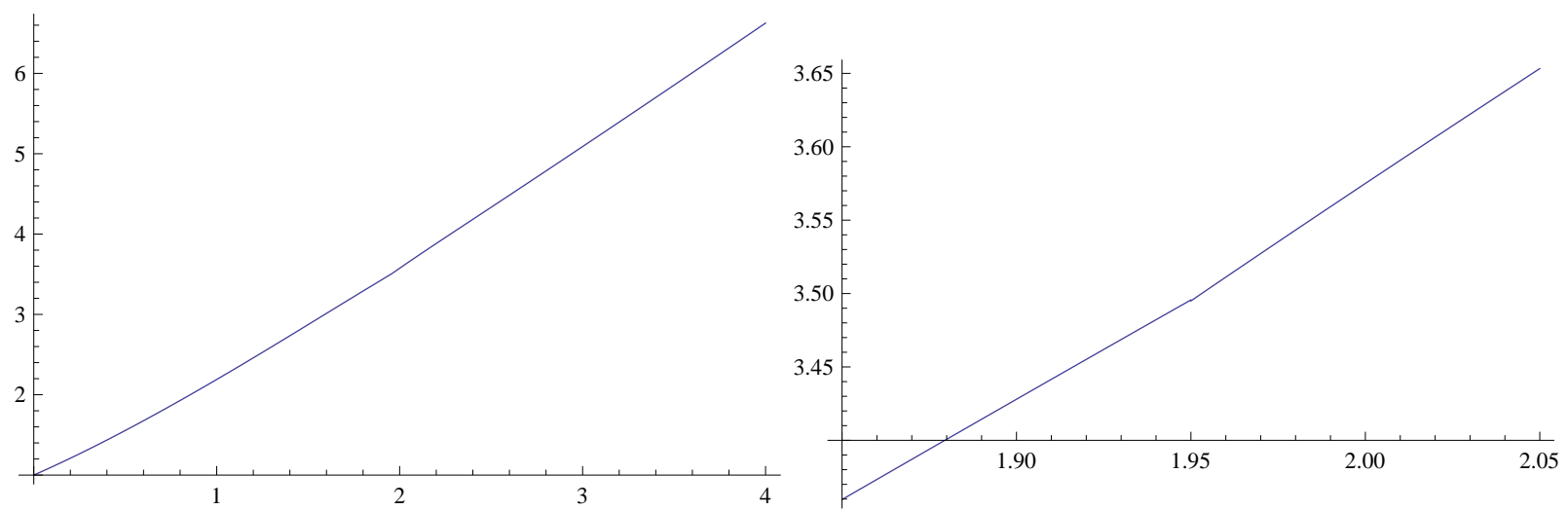

FIG. 2: Matching the expansions (C23) and (C24). The region near $x=1.95$ is enlarged in the right figure.

and finally we obtain

$$
\begin{aligned}
\sum_{n, m} \ln \left(\lambda_{n, m}^{-1 / 2}\right) & =-\sum_{n} \frac{\pi n a}{2 b} \int_{0}^{\infty} \mathrm{d} x\left[1-x f^{\prime}(x) / f(x)\right] \\
& =\frac{\pi}{24} \frac{a}{b} \int_{0}^{\infty} \mathrm{d} x\left[1-x f^{\prime}(x) / f(x)\right]
\end{aligned}
$$

For an $T \times R$ rectangle with $T \gg R$, when

$$
\lambda_{n, m}=\frac{\pi^{2} n^{2}}{R^{2}}+\frac{\pi^{2} m^{2}}{T^{2}} \quad \text { rectangle }
$$

resulting in

$$
f(x)=\sqrt{1+x^{2}} \quad \text { rectangle, }
$$

we reproduce the well-known result for the Lüscher term by the substitution $a=T / \pi$, $b=R / 2$.

For an outstretched ellipse we have from the expansion (C9b)

$$
f(x) \stackrel{x \leq x_{0}}{=} 1+x+\frac{x^{2}}{4}-\frac{x^{3}}{16}-\frac{x^{4}}{128}+\frac{15 x^{5}}{1024}-\frac{9 x^{6}}{2048}+\ldots
$$

for $x<x_{0} \approx 1.95$, where the series converges, and

$$
\begin{aligned}
f(x) \stackrel{x>x_{0}}{=} & 1.600095 x+\frac{0.960520}{x}-\frac{1.041899}{x^{3}}+\frac{2.418227}{x^{5}}-\frac{7.138640}{x^{7}}+\frac{23.73152}{x^{9}} \\
& -\frac{84.70585}{x^{11}}+\ldots
\end{aligned}
$$

for $x>x_{0} \approx 1.95$. They match each other pretty well, as is depicted in Fig. 2. The numerical 
value of the integral on the right-hand side of Eq. (C20) is then 2.84 to be compared with $\pi$ as is expected from Eq. (51).

To clarify the calculation, let us keep only three terms on the right-hand side of Eq. (C9b), when we get a quadratic equation and analytical formulas are available. We then find

$$
f(x)=x+\sqrt{1+x^{2} / 2}
$$

and expanding it either in $x$ or in $1 / x$ we obtain analogs of Eqs. (C23) and (C24). The two expansions now match at $x_{0}=\sqrt{2}=1.41$ to be compared with $x_{0}=1.95$ for all nine terms left on the right-hand side of Eq. (C9b). The value of the integral is now $\sqrt{2} \log [3+2 \sqrt{2}]=2.49$ which is smaller than 2.84 , which may characterize to which extend the approximation of $\alpha_{m}$ by the eight term in $(\mathrm{C} 9 \mathrm{~b})$ is better than by the tree terms. In Appendix D we confirm the value of $\pi$ by another method.

\section{Appendix D: Lüscher term from conformal anomaly}

A starting point is the formula $[7,19]$

$$
\text { Lüscher term }=\frac{1}{96 \pi} \int \mathrm{d} \tau \mathrm{d} \sigma \partial_{a} \ln \rho \partial_{a} \ln \rho
$$

that relates the Lüscher term to the conformal anomaly. Here the metric $\rho$ is

$$
\rho=\left|\frac{\mathrm{d} z}{\mathrm{~d} u}\right|^{2}, \quad u=\tau+\mathrm{i} \sigma
$$

where

$$
\omega=h \cosh (\tau+\mathrm{i} \sigma)
$$

runs inside the ellipse with $\tau$ and $\sigma$ being elliptic coordinates as in Eq. (C2). The function (inverse to (B23) from [6])

$$
z(\omega)=\sqrt{\nu} \operatorname{sn}\left(\frac{2 K}{\pi}\left(\frac{\pi}{2}-\sigma+\mathrm{i} \tau\right)\right)=\sqrt{\nu} \operatorname{sn}\left(K-\frac{2 K}{\pi}(\sigma-\mathrm{i} \tau)\right)=\sqrt{\nu} \frac{\operatorname{cn}\left(\frac{2 K}{\pi}(\sigma-\mathrm{i} \tau)\right)}{\operatorname{dn}\left(\frac{2 K}{\pi}(\sigma-\mathrm{i} \tau)\right)}
$$

conformally maps ellipse onto a unit disk.

To calculate (D2) for $a / b \gg 1$, we substitute

$$
\frac{\operatorname{cn}\left(\frac{2 K}{\pi} \mathrm{i} u, \nu\right)}{\operatorname{dn}\left(\frac{2 K}{\pi} \mathrm{i} u, \nu\right)}=\frac{1}{\operatorname{dn}\left(\frac{2 K}{\pi} u, \nu^{\prime}\right)}
$$


with

$$
\nu^{\prime}=\sqrt{1-\nu^{2}} \rightarrow 0
$$

Differentiating, we get

$$
\frac{\mathrm{d} z}{\mathrm{~d} u}=\sqrt{\nu} \frac{2 K}{\pi}\left(1-\nu^{2}\right) \frac{\operatorname{sn}\left(\frac{2 K}{\pi} u, \nu^{\prime}\right) \operatorname{cn}\left(\frac{2 K}{\pi} u, \nu^{\prime}\right)}{\operatorname{dn}^{2}\left(\frac{2 K}{\pi} u, \nu^{\prime}\right)} .
$$

Using the fact that Jacobi elliptic functions reduce to trigonometric functions as $\nu^{\prime} \rightarrow 0$ and substituting

$$
K \stackrel{\nu \rightarrow 1}{\rightarrow} \frac{\pi^{2}}{8} \frac{a}{b}
$$

we infer from Eq. (D7)

$$
\frac{\mathrm{d} z}{\mathrm{~d} u} \propto \exp \left(-\mathrm{i} \frac{\pi}{2} \frac{a}{b} u\right) \quad \Longrightarrow \quad \rho \propto \exp \left(\pi \frac{a}{b} \sigma\right)
$$

and

$$
\text { Lüscher term }=\frac{1}{96 \pi} \int_{0}^{\tau_{0} \approx \frac{b}{a}} \mathrm{~d} \tau \int_{0}^{2 \pi} \mathrm{d} \sigma\left(\pi \frac{a}{b}\right)^{2}=\frac{\pi^{2}}{48} \frac{a}{b}
$$

that confirms the extra $\pi / 2$ in Eq. (51) for an ellipse in comparison with a rectangle.

[1] J. Douglas, Solution of the problem of Plateau, Trans. Am. Math. Soc. 33, 263 (1931).

[2] A. G. Cohen, G. W. Moore, P. C. Nelson, and J. Polchinski, An off-shell propagator for string theory, Nucl. Phys. B 267, 143 (1986).

[3] A. M. Polyakov, Talk at the Workshop "Particles, Fields and Strings", Vancouver, July 1997, unpublished.

[4] A. A. Migdal, Loop equation and area law in turbulence, Int. J. Mod. Phys. A 9, 1197 (1994) [arXiv:hep-th/9310088].

[5] V. S. Rychkov, Wilson loops, D-branes, and reparametrization path integrals, JHEP 0212, 068 (2002) [arXiv:hep-th/0204250].

[6] Y. Makeenko and P. Olesen, Wilson loops and QCD/string scattering amplitudes, Phys. Rev. D 80, 026002 (2009) [arXiv:0903.4114 [hep-th]].

[7] M. Lüscher, K. Symanzik and P. Weisz, Anomalies of the free loop wave equation in the WKB approximation, Nucl. Phys. B 173, 365 (1980); M. Lüscher, Symmetry breaking aspects of the roughening transition in gauge theories, Nucl. Phys. B 180, 317 (1981). 
[8] J. Ambjørn, P. Olesen and C. Peterson, Observation of a string in 3-dimensional lattice gauge theory, Phys. Lett. B 142, 410 (1984); Ph. De Forcrand, G. Schierholz, H. Schneider, M. Teper, The string and its tension in SU(3) lattice gauge theory, Phys. Lett. B 160, 137 (1985); M. Lüscher and P. Weisz, Quark confinement and the bosonic string, JHEP 0207, 049 (2002) [arXiv:hep-lat/0207003].

[9] W. Hofstetter and W. Zwerger, Single-electron box and the helicity modulus of an inverse square XY model, Phys. Rev. Lett. 78, 3737 (1997).

[10] P. Buividovich and Y. Makeenko, Path integral over reparametrizations: Levy flights versus random walks, arXiv:0911.1083 [hep-th].

[11] I. S. Gradshteyn and I. M. Ryzhik, Tables of integrals, series, and products, (5ed., AP, 1996)

[12] Z. Jaskolski and K. A. Meissner, Static quark potential from the Polyakov sum over surfaces, Nucl. Phys. B 418, 456 (1994) [arXiv:hep-th/9302100].

[13] M. Abud, C. G. Bollini and J. J. Giambiagi, Quantized Wilson loops with dimensional regularization, Nucl. Phys. B 204, 109 (1982).

[14] B. Durhuus, P. Olesen and J.L. Petersen, On the static potential in Polyakov's theory of the quantized string, Nucl. Phys. B 232, 291 (1984).

[15] Y. Makeenko and P. Olesen, Implementation of the duality between Wilson loops and scattering amplitudes in QCD, Phys. Rev. Lett. 102, 071602 (2009) [arXiv:0810.4778 [hep-th]].

[16] N. W. McLachlan, Theory and application of Mathieu functions, Oxford (1947).

[17] F. A. Alhargan, A complete method for the computation of Mathieu characteristic numbers of integer order, SIAM Review 38, 239 (1996).

[18] O. Alvarez, Static potential in string theory, Phys. Rev. D 24, 440 (1981).

[19] H. W. J. Blöte, J. L. Cardy and M. P. Nightingale, Conformal invariance, the central charge, and universal finite size amplitudes at criticality, Phys. Rev. Lett. 56, 742 (1986); I. Affleck, Universal term in the free energy at a critical point and the conformal anomaly, Phys. Rev. Lett. 56, 746 (1986). 